\title{
Development of ergonomic website for engineering education
}

\author{
Arnes Faradilla ${ }^{1}$, and Muhammad Ridwan Andi Purnomo $^{2}$ \\ ${ }^{1}$ Industrial Engineering of Universitas Trisakti, Jakarta, Indonesia \\ ${ }^{2}$ Industrial Engineering of Universitas Islam Indonesia, Yogyakarta, Indonesia
}

\begin{abstract}
Engineering education contains two aspects; theory and practice. Practice activity supports the students to implement the theory obtained in the class. Therefore, it will be better if the theory has material visualization in order to support and make easier the student and lecturer in teaching activity. Analisis Perancangan Kerja dan Ergonomi (APK \& E) is one of the laboratories in Industrial Engineering, Universitas Islam Indonesia. APK \& E laboratory which uses website as a tool to communicate to the students. Currently, the existing website still show standard display by conventional way. Based on the questionnaire, the student difficult to understand the material especially for Micromotion Study since this material requires the video to show the student in assembly activity. Augmented Reality (AR) combines the real and virtual objects that interactive in the real time and registered in $3 \mathrm{D}$ space. AR also combines the real and virtual in displaying information in the real time, in a way that enhances the individual abilities to operate the system in the real world. The system which combines between AR and Micromotion Study was come up. The required software to develop the online AR system is FLARToolKit 2.5.1. In this research, marker detection accuracy measurement of the used toolkit has been conducted and it showed $85 \%$ of accuracy level.
\end{abstract}

\section{Introduction}

Engineering education contains two aspects; theory and practice. Nowadays, theory is not enough anymore if there is no practice of material inside. Therefore, the theory is needed to visualize the material in order to support the student and lecturer in teaching activity. The APK \& E is one of the laboratories in Industrial Engineering which concerns in analysis of work design and ergonomics. APK \& E Laboratory which uses website as a tool to communicate to the students. Currently, the APK \& E lab work contains 6 subjects; Anthropometrics, Micromotion study, Biomechanics, Physical Work Environment, Physiology and Work Sampling. The APK \& E Laboratory provides lab work subject in the website and updated every week continuously. In fact, the website just show standard display and conventional. The provided modules are still standard and not attractive. Based on the preliminary questionnaire, the weaknesses of APK \& E website are color combination as much as $21.6 \%$, website's display as much as $18.9 \%$, picture as much as $13.5 \%$, font's type as much as $13.5 \%$, animation as much as $13.5 \%$, assembly visualization on the web as much as $13.5 \%$ and the other weaknesses as much as $5.4 \%$.

There is one subject of lab work, which is Micromotion study, which requires a video to show an example of assembly activity conducted in the lab work to increase the student understanding. Currently, the students just download the module without understand the content leads to inefficient study time in the class. The assistant must explain all over again the subject because the students have not understood the material.

AR combines the real and virtual that interactive in the real time and registered in 3D space. AR combines the real and virtual in displaying information in the real time, in a way that enhances the individual abilities to operate the system in the real world. Based on the problem, the system to combine between AR and Micromotion Study module was come up. In this research, FLARToolKit will be utilized to develop the online AR system.

\section{Literature Review}

Reinhart \& Patron (2003) have discussed the integrating Augmented Reality into assembly domain. AR is a new technology of human-machine interaction based on the computers that show the information of a real world. The information is shown on display. AR can increase the worker understanding in virtual object based on the real condition. The functions of AR in assembly domain are guiding the assembly worker and as the visual assembly instruction. Siltanen (2012) stated that marker-based systems have many advantages in such cases, as we justify later in this work. In consequence, the author's main contribution is in marker-based applications. Often, the ultimate goal is a mobile solution, even though the demonstration may run on a PC environment. Hence, the focus is on methods that require little processing capacity 
and little memory. Ong (2011) present the augmented assembly technology based on the 3D bare-hand interaction. Augmented reality was applied into augmented assembly. AR is required to display the information and interaction between the environment and the users. This paper presents the 3D bare hand interaction in an augmented assembly environment to manipulate and assembly the virtual components. Dangelmaier et al (2004) describe the Virtual and Augmented Reality Support for Discrete Manufacturing System Simulation. The purpose of this research is because the dynamics of the innovation is increase and the product life cycle becomes shorter. Therefore, they use the digital factory/virtual production method to reduce the time and cost. The result of this research is a general application of the VR and AR-technologies and to realize the simulation by developing the appropriate link and the integration mechanism.

Tateno et al (2005) explain about the nested for Augmented Reality. They require camera calibration in developing the marker. After design the marker, then develop the AR system to demonstrate the ability of the nested marker. It requires the ARToolKit framework as software. The purposes of this research are visualize the marker design and the experiment evaluation using artificially rendered marker images.

Alkhamisi and Monowar (2013) explain the prospect of AR is used to facilitate the learning whereas it enables students to access location, specific information provided through various sources.

Domingues et al (2010) describe the virtual laboratories in (bio) chemical engineering education. This paper describes the design and the implementation of two virtual labs for biochemical engineering education intended for students at the BSc degree. The effectiveness of the implemented system was evaluated through direct experimentation and survey (through questionnaires) with students taking the chemical technology lab course. The survey results show the value of $93 \%$ students consider the virtual labs of great utility.

Khapre \& Chandramohan (2011) present personalized web selection. They describe the display selection on the web appropriate with people's preferences. In this paper, they present an extensive study of different selection techniques towards advanced personalization of the web in service selection.

\section{Research Methodology}

In this research, there are several steps in developing the online AR system and measuring the successful test. The first step of this research is identifying the problem of APK \& E Laboratory. It was obtained from questionnaire according to student understanding problem. The second step is creating the video objects to be visualized in the online AR system. The well known 3D graphic design software called 3D Studio Max 9 is utilized. The reason of using the 3D Studio Max software is because the software can also be used to create an animation of the assembly process of T-shape electrical plug based on the built 3D objects. The third step is creating the proper unique marker for the online AR system. The software to be used to design a proper marker for the online AR system is Adobe Photoshop. The next step is developing the online AR system using FLARToolKit 2.5.1. After that, testing the online AR system. First step in testing the online AR system is testing a marker which can display the related video in the virtual environment. The second step is rotaring the marker in $\mathrm{Y}$ axis and $\mathrm{Z}$ axis. The online $\mathrm{AR}$ system to be deployed directly to the internet.

\section{AR System Development}

Technically, the aim of developing an online AR system is to visualize the video objects in a web-based virtual environment using unique markers. Basically, when the user accesses the web page, the AR system will be downloaded automatically to the user computer as activeX object. The activeX object will access webcam installed in the user computer to get real time picture of the user. The user needs to show a printed marker to the webcam in order to see the virtual object in the virtual environment. The detail step of developing the AR system will be explained as follows:

1. Creating the Video Objects

Based on the questionnaire, the selected object of assembly activity is T-shape electrical plug. The video objects to be visualized are the video on T-shape electrical plug assembly process, measurement activity, initial layout of assembly work station and suggested layout of assembly work station.

2. Creating the Marker

The function of marker is to trigger related video appearance in virtual environment. The software to be used to design a marker is Adobe Photoshop. The aim of using Adobe Photoshop is because the software can be used to create the proper marker and also to determine the pixel size in each marker. The first step in creating the marker is designing a rectangle marker with $2 \mathrm{~cm}$ width black frame. The second step is creating an image with $4 \mathrm{~cm}$ in the middle of marker. In this research, there are 4 designed marker with picture in the middle represents the video object to be displayed in the virtual environment.

3. Development of Marker Detection Module

The steps in development of marker detection module are finding potential marker, marker pattern identification and matching found marker with database of marker. The detail step in the development of marker detection module will be explained as follows:

a. Finding the Potential Marker

The first step in finding the potential marker is doing the grayscaling of the original image. There is a quite contrast object; a black bordered on white paper. Thus, most probably a good direction is to search for black image frame surrounded by white areas and do their analysis. The second step is to try doing thresholding for finding the black image frame. One of method to be used to threshold the black image frame is Otsu method.

b. Marker Pattern Identification 
The first step of marker pattern identification is the image marker divided into cells. The marker image is divided by dark gray lines of cells in the same width and height. The second step is counting the number of white pixels in each cell. If the number is greater than cell's area then assumed that the cell is filled by white color which corresponds to "1". And if the number is less than half of the cell's area then the black cell will correspond to "0".

c. Matching Found Marker with Data Base of Marker The finding a matching marker's database needs to do compares of extracted marker data with every object in the database - compare the possible rotations of extracted object data with the database. The required FLARToolKit is saved in ".dat" file.

4. Pseudocode

The language to be used to explain the process of developing program based on natural language is pseudocode. The pseudocode of this system is shown in Figure 2.

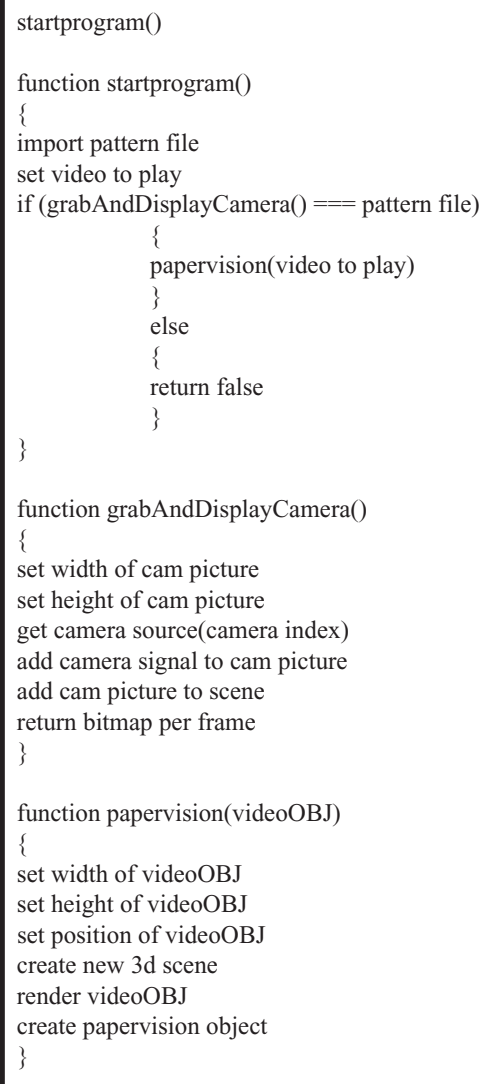

Figure 2. Pseudocode

Based on the pseudocode above, the first step is starting to initialize the program with importing pattern file and set the video to be played. After that, set the dimension of bitmap file to be captured from camera and get source the camera to grab the scene per frame and add to display. The output from this step will be an image file to be compared with pattern file. If it is similar (Boolean value return true), then it will go to create paper vision object. The first step was to set the dimension of the video that mentioned in previous, and then set the position video object. The last step was created the $3 \mathrm{~d}$ scene and renders the object.

5. Testing the AR System

Basically, the video's position can be controlled using marker. The aim of controlling the video objects using marker is in order to show the video objects in various angles. The screenshot of video in normal position is shown in Figure 3.

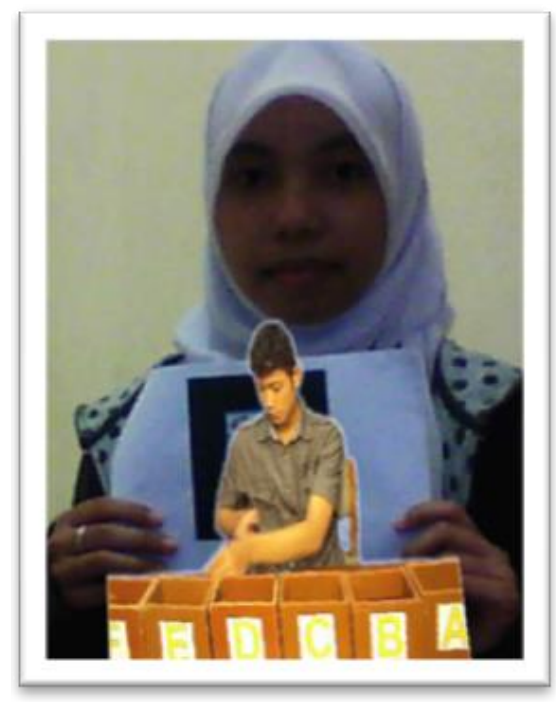

Figure 3. Video in Normal Position

When the marker turned $45^{\circ}$ with $\mathrm{Y}$ axis as center, thus video will rotate $45^{\circ}$. The screenshot of rotated video in AR system is shown in Figure 4 and Figure 5.

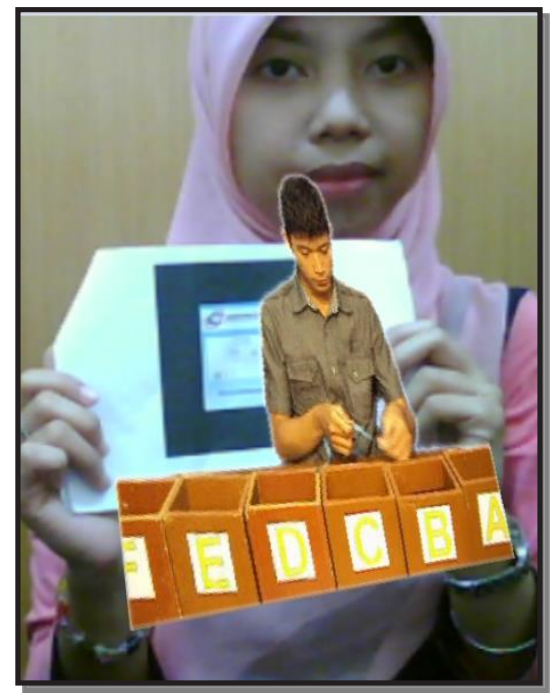

Figure 4. Rotated Video in $\mathrm{Y}$ axis as far as $+45^{\circ}$ 


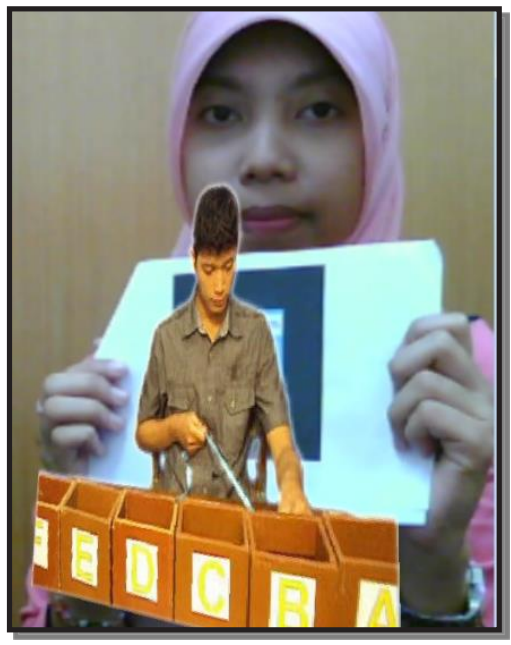

Figure 5. Rotated Video in $\mathrm{Y}$ axis as far as $-45^{\circ}$

When the marker turned $45^{\circ}$ with $\mathrm{Z}$ axis as center, thus video will rotate $45^{\circ}$. The screenshot of rotated video in AR system is shown in Figure 6 and Figure 7.

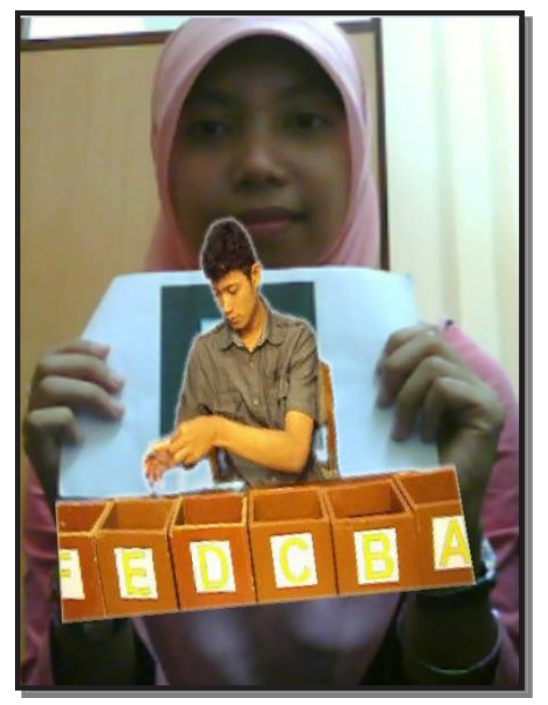

Figure 6. Rotated Video in $\mathrm{Z}$ axis as far as $+45^{\circ}$

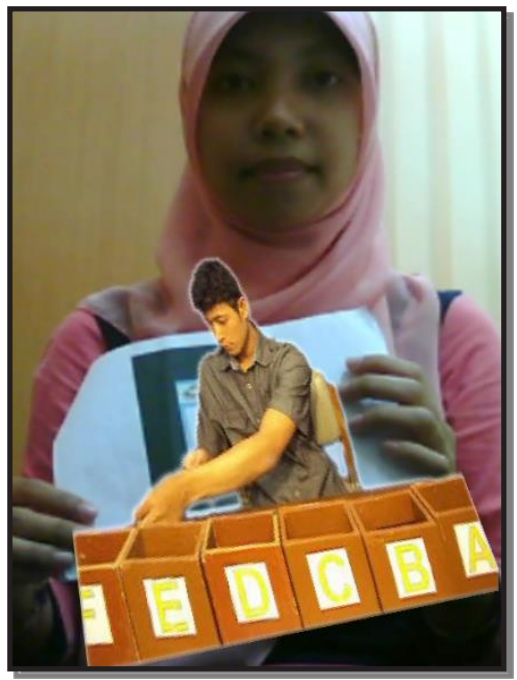

Figure 7. Rotated Video in $\mathrm{Z}$ axis as far as $-45^{\circ}$

\section{Discussion}

The next step is measuring the marker detection accuracy of the used toolkit. The marker detection accuracy is needed since marker has function to trigger the related video in the virtual environment. The detail step of measuring the marker detection accuracy of the used toolkit will be explained as follows:

1. Noising the Marker

In this step, the required data is collecting the marker which is able to show the video in the online AR system. Noise is a disturbance of brightness in the image. Marker to be given the noise in various sizes. The aim of giving the noise in the marker is to measure the ability of marker contained noise in detecting the object. The formula to measure the noise in the marker is shown in Equation 1.

$\mathrm{A}=\mathrm{WxH}$

Where:

$\mathrm{A}=$ Area of marker/noise $\left(\right.$ pixel $\left.^{2}\right)$

$\mathrm{W}=$ Width of marker/noise ( pixel )

$\mathrm{H}=$ Height of marker/noise ( pixel )

There are 5 treatments in noising the marker. The 5 treatments as follows:

a. In the first treatment, there is no noise in marker. The size of whole marker is 800 pixels for width and 1000 pixels for height. The result of calculation is $2,500,000$ pixels $^{2}$

b. In the second treatment, the noise's size is 100 pixels for width and 190 pixels for height. Based on the result, camera still read the marker and is able to visualize the video. The result of the second treatment is 19,000 pixels $^{2}$

c. In the third treatment, the noise's size is 200 pixels for width and 340 pixels for height. Based on the result, camera still read the marker and is able to visualize the video. The result of the third treatment is 68,000 pixels $^{2}$

d. In the fourth treatment, the noise's size is 340 pixels for width and 400 pixels for height. Based on the result, camera still read the marker and is able to visualize the video but in weak detection. The result of the fourth treatment is 136,000 pixels $^{2}$

e. In the fifth treatment, the noise's size is 500 pixels for width and 420 pixels for height. Based on the result, camera still read the marker and is able to visualize the video but in the weak detection. The result of the fifth treatment is 210,000 pixels $^{2}$

f. In the sixth treatment, the noise's size is 750 pixels for width and 500 pixels for height. Based on the result, camera cannot read the marker and visualize the video. The result of the sixth treatment is 385,000 pixels $^{2}$

2. Accuracy Level of Marker

The accuracy level of marker is measuring the capability level of marker in detecting the video and also to measure the noise level in order to create a new marker. It can be determined by testing the noise of 
each marker. The accuracy level of marker can be determined by calculating the percentage of each treatment. The formula to calculate the accuracy level of marker is shown in Equation 2.

$$
\mathrm{ND}=\frac{\mathrm{AN}}{\mathrm{AM}} \times 100 \%
$$

Where :

ND $=$ Noise Detection (Percentage)

$\mathrm{AN}=$ Area of Noise (Pixel)

$\mathrm{AM}=$ Area of Marker (Pixel)

Based on the result above, the accuracy level of the used toolkit has $85 \%$. It means that webcam still able to visualize the video which has marker contained noise less than $15 \%$. Thus, the marker can be varied if marker value higher than $15 \%$.

\section{Conclusion and Suggestion}

The result of this research can be concluded as follows:

a. Conclusion

1. The developed AR system is able to visualize Micromotion Study module.

2. The accuracy level of marker is $85 \%$. It means that system can read the marker with noise less than $15 \%$.

b. Suggestion

1. For the next research can use dynamic threshold to decrease the noise level because it can reduce the noise level.

2. Develop further research for another APK \& E subjects using AR technology in order to make understand better of the students.

\section{References}

1. G. Reinhart, C. Patron. Integrating augmented reality in the assembly domain - fundamentals, benefits and applications. Proceeding of TU Munchen, Garching. Germany. (2003).

2. S. Siltanen. Theory and applications of maker-based augmented reality. VTT Science 3. (2012).

3. W. Ong. Augmented assembly technologies based on 3D bare-hand interaction. National University of Singapore 11757. Singapore. (2011).

4. Dangelmaier et al. Virtual and augmented reality support for discrete manufacturing system simulation. Computers in Industry 56: 371-383. (2004).

5. K. Tateno. I. Kitahara, Y. Ohta. A nested marker of augmented reality. Proceedings of the 2nd International Workshop on Augmented Reality. Japan. (2005).

6. A.O. Alkhamisi, M.M. Monowar. Rise of Augmented Reality : Current and Future Application Areas. International Journal of Internet and Distributed Systems. 25-34. (2013).

7. Domingues et al. Virtual laboratories in (bio)chemical engineering education. IBB-Institute for Biotechnology and Bioengineering, Centre of Biological Engineering, Universidade do Minho, Campus de Gualtar. Portugal. (2010).

8. Khapre, Chandramohan. Personalized web service selection. Department of Computer Science, Pondicherry University, Pondicherry. India. (2011) 